\title{
Chemical Etching and Surface Oxidation Studies of Cadmium Zinc Telluride Radiation Detectors
}

M.E Özsan ${ }^{* 1}$, P.J. Sellin ${ }^{1}$, P. Veeramani ${ }^{1}$, S.J. Hinder ${ }^{2}$, M.L.T. Monnier ${ }^{2}$, G. Prekas ${ }^{1}$, A. Lohstroh ${ }^{1}$, M. A. Baker ${ }^{2}$

${ }^{1}$ Department of Physics, Faculty of Engineering and Physical Sciences, University of Surrey, Guildford, GU2 7XH, UK.

${ }^{2}$ The Surface Analysis Laboratory, Faculty of Engineering and Physical Sciences, University of Surrey, Guildford, GU2 7XH, UK

\begin{abstract}
:
CdZnTe (CZT) is commonly used as a radiation detector material and the surface properties are important as they influence detector performance. The surface chemistry is generally controlled through chemical etching and oxidation processes. In this paper, XPS is employed to investigate changes in the surface composition of single crystal $\mathrm{Cd}_{0.95} \mathrm{Zn}_{0.05}$ Te samples after exposure to bromine in methanol (BM) chemical etching treatments and subsequent oxidation in air or $30 \% \mathrm{H}_{2} \mathrm{O}_{2}$. $\mathrm{BM}$ treatment of CZT is found to result in a graded Te-rich surface layer which increases in thickness as a function of BM concentration. Room temperature air oxidation of $0.2 \%$ BM treated CZT follows a logarithmic rate law. BM treated CZT exposed to 30 $\% \mathrm{H}_{2} \mathrm{O}_{2}$ for 30 seconds shows a linear increase in the $\mathrm{TeO}_{2}$ oxide thickness with increasing BM concentration up to a BM concentration of $1.5 \%$. Above $2 \% \mathrm{BM}$ concentration, the Te enrichment in the CZT surface region has reached saturation and is effectively pure Te.
\end{abstract}

Keywords: XPS, CdZnTe, radiation detectors, oxidation.

"Corresponding author: Dr M. Ersin Özsan, Email: m.ozsan@surrey.ac.uk, 


\section{Introduction}

In this paper a study of the chemical etching and oxidation processes influencing the surface properties of $\mathrm{CdZnTe}(\mathrm{CZT})$ radiation detectors is presented. CZT with desirable properties has actively been developed for room temperature $\mathrm{X}$ ray and gamma-ray solid-state radiation detectors with high sensitivity and good resolution for $10-500 \mathrm{keV}$ photons $(1,2)$. Applications range from medical imaging to environmental protection. The surface properties play an important role in the performance of planar or pixellated CZT detectors when devices are fabricated with metals electrodes, e.g., Pt, Au. Chemical etching has often been used to remove damage caused by ingot slicing, dicing and mechanical polishing in order to produce smooth and defect-free surfaces. Bromine in methanol (BM) is one of the most common chemical etchants used to clean CZT surfaces but it is known to produce non-stoichiometric and especially Te-rich, low resistivity surfaces, causing surface leakage currents detrimentally affecting the performance of CZT detectors (3-7).

Various groups have reported methods for effective passivation processes to overcome the surface leakage currents which involve producing tellurium oxide, $\mathrm{TeO}_{2}$ on the CZT surface. Such passivation processes generate a thin $(2.0-4.0 \mathrm{~nm})$ $\mathrm{TeO}_{2}$ layer which renders the surface electrically and chemically inert to its environment. Chen et al used an aqueous solution of hydrogen peroxide $\left(\mathrm{H}_{2} \mathrm{O}_{2}\right)$ to oxidise the CZT surface and produce a thin $\mathrm{TeO}_{2}$ layer (8). Recently, XPS studies carried out on CZT surfaces chemically etched with 5\% BM showed $2.0 \mathrm{~nm}$ thick Terich layers form independent of the etch time or solution acidity (9). It was also mentioned that Te-rich surfaces oxidize rapidly in air, however the extent or details of this air oxidation of CZT surfaces were not discussed.

In this paper, we present an X-ray photoelectron spectroscopy (XPS) study of changes in the CZT surface composition and surface oxidation as a result of BM etching and subsequent oxidation in air or $30 \% \mathrm{H}_{2} \mathrm{O}_{2}$. 


\section{Experimental procedure}

\subsection{Surface preparation}

The CZT single crystals used in this study were obtained from two sources, Durham University, UK and Yinnel Tech Inc., USA, grown using multi-tube physical vapour transport and the modified vertical Bridgman methods respectively. The $\mathrm{Zn}$ concentration for both materials was approximately 5 at.\% (determined by photoluminescence (10) and in both cases the CZT crystals exhibited high resistivities of $10^{9}-10^{10}$ ohm-cm. The $\mathrm{Cd}_{0.95} Z_{0.05}$ Te samples used for the XPS surface studies had dimensions of $8 \times 8 \times 3 \mathrm{~mm}^{3}$. Surfaces of samples were prepared and analysed using a standard procedure that involved mechanically polishing the sample surfaces with $\mathrm{Al}_{2} \mathrm{O}_{3}$ powder down to $0.05 \mu \mathrm{m}$ followed by chemical etching in freshly prepared BM solution. To investigate the influence of BM concentration on surface composition, after BM exposure, the samples were rinsed in methanol, dried with $\mathrm{N}_{2}$ and immediately transferred into the UHV XPS equipment.

The oxidation experiments involved freshly etching the samples in BM followed by exposure in laboratory air or in an aqueous $30 \% \mathrm{H}_{2} \mathrm{O}_{2}$ solution. For the air oxidation work, the surface was initially analysed after BM etching and oxidation monitored by repeated sequences of air exposure followed by insertion into the XPS spectrometer. For oxidation in $\mathrm{H}_{2} \mathrm{O}_{2}$, the sample was etched in different $\mathrm{BM}$ concentrations followed by a 30 second dip in $30 \% \mathrm{H}_{2} \mathrm{O}_{2}$. These experiments were performed on the same CZT sample. After each exposure and analysis, the surface was mechanically re-polished.

\subsection{X-ray Photoelectron Spectroscopy}

XPS analysis was undertaken using a Thermo Scientific (East Grinstead, UK) Theta Probe spectrometer, enabling Angle Resolved X-ray Photoelectron Spectroscopy (ARXPS) analysis. In this work the microfocus monochromated Al Ka source $\left(\mathrm{hv}=1486.6 \mathrm{eV}\right.$ ) was used and area of $0.5 \mathrm{~mm}^{2}$ analysed. High resolution, core level spectra were acquired in both the standard and the angle resolved modes of operation with a pass energy of $150 \mathrm{eV}$. Quantification of the spectra was 
performed following the removal of a non-linear (Shirley) background and the Thermo Avantage software incorporates transmission function corrected Wagner sensitivity factors. XPS depth profiles have been performed using either an $\mathrm{Ar}^{+}$ion gun operating at $3 \mathrm{kV}$ or by non-destructive Angle Resolved XPS employing Thermo's ARProcess software to construct angle resolved depth profiles.

\section{Results and discussion}

Fig. 1 (a) shows the Te 3d XPS spectrum from a CZT surface etched in $2 \%$ BM and Fig.1 (b) is the same surface after subsequent passivation in $30 \%$ aqueous $\mathrm{H}_{2} \mathrm{O}_{2}$ solution for 2 minutes. Exposure to the $2 \%$ BM shows the $\mathrm{Te} 3 \mathrm{~d}_{5 / 2}$ peak at a binding energy of $572.9 \mathrm{eV}$, attributable to a Te-rich CZT surface. No Te oxide peak can be observed. $\mathrm{H}_{2} \mathrm{O}_{2}$ dipped samples after $2 \% \mathrm{BM}$ etching, show the formation of $\mathrm{TeO}_{2}$ at $576.6 \mathrm{eV}$. The Te oxide layer thickness on CZT was estimated from the intensity of the $\mathrm{Te} 3 \mathrm{~d} \mathrm{CZT}$ surface and $\mathrm{TeO}_{2}$ oxide peaks, labelled $I_{\text {Met }}$ and $I_{\text {ox }}$ respectively, using the equation (11);

$$
d_{o x}=\lambda_{o x} \cos \theta \ln \left(\frac{I_{o x}}{I_{M e t}}+1\right)
$$

Where, $\lambda_{o x}$ is the attenuation length which is calculated to be $2.63 \mathrm{~nm}$ (based upon the relationship given by Seah and Dench (12)) and $\theta$ is the photoelectron take-off angle with respect to the surface normal. The thickness of the $\mathrm{TeO}_{2}$ layer on the $\mathrm{Cd}_{0.95} \mathrm{Zn}_{0.05}$ Te sample shown in Fig.1 (b) was calculated to be $2.3 \mathrm{~nm}$.

The effect of BM etching concentration on the CZT surface composition was investigated by $\mathrm{Ar}^{+}$ion sputter depth profiling. The mechanically polished CZT sample was used for three sequential experiments. Firstly, depth profiling was performed on the mechanically polished surface, then the sample was re-polished and freshly etched in $0.2 \%$ BM prior to the second analysis, finally the sample was again re-polished and etched in 1.0\% BM. The results obtained for the relative atomic percentages for Te $3 d_{5 / 2}, C d 3 d_{5 / 2}$ and $Z n 2 p_{3 / 2}$ as a function of $\mathrm{Ar}^{+}$ion etch time are shown in Fig. 2. The mechanically polished sample showed a near 
stoichiometric surface composition, whereas the BM etched samples showed Te-rich surfaces and a graded composition in the near surface region. The Te-rich graded region for the $1.0 \% \mathrm{BM}$ is nearly double the thickness of the $0.2 \% \mathrm{BM}$ etched sample.

Fig. 3 shows the variation of Te $3 d_{5 / 2}$ peak energy as a function of angle for a cleaved CZT sample and CZT samples exposed to $0.5 \%, 1 \%$ and $5 \% \mathrm{BM}$ treatments. In this plot, the higher analysis angles correspond to a more surface sensitive analysis than the lower angles. The cleaved sample shows a Te $3 d_{5 / 2}$ binding energy which varies little (572.60 and $572.75 \mathrm{eV}$ ) between the high and low angles, as would be expected. This binding energy is in good agreement with that of Etcheberry et al for bulk CZT (13). The 0.5\% and 1\% BM treatments show surfaces where there is a clear trend for a higher binding energy to be observed near the surface and the binding energy to progressively drop as the analysis penetrates further into the bulk. In both the $0.5 \%$ and $1 \%$ BM treatments, there is a drop of between 1.5 and $2.0 \mathrm{eV}$ between the most surface sensitive to most bulk sensitive analysis, evidence again of a graded stoichiometry between the surface and bulk. However, for a 5\% BM treatment, there is no change in binding energy as a function of depth and the binding energy remains at an average value of $573.15 \mathrm{eV}$, attributable to metallic Te $(14,15)$. The depth profiles in Fig. 2 show that the Te-rich region increases in thickness as a function of $\mathrm{BM}$ concentration. Hence, for a $5 \%$ BM treatment, the invariant Te $3 d$ binding energy is evidence that the thickness of the Te-rich graded region is sufficiently large, that a negligible drop in Te concentration is observed over the XPS analysis depth.

Air oxidation of CZT surfaces has been investigated with a view to understanding the oxidation process in more detail. A CZT sample was freshly etched in $0.2 \% \mathrm{BM}$ etch and the oxide thickness plotted as a function of time in Figure 4. Initially, rapid oxide growth is observed, but it then slows down. The oxide reaches a thickness of $1.3 \mathrm{~nm}$ after 2200 hours. Consequently, even though a Terich surface has been developed through the $0.2 \% \mathrm{BM}$ etch, the air-oxidation process of CZT is very slow compared to other technologically important metals and semiconductors, e.g. Ti, Al, Si etc. Low temperature oxidation often obeys a logarithmic rate law and a best fit to the data was obtained using the equation 2 : 


$$
\boldsymbol{d}=0.285 \ln (0.047 \boldsymbol{t}+0.987)
$$

where $\mathrm{d}$, is the oxide thickness and $\mathrm{t}$, the exposure time.

It is well-known that $\mathrm{H}_{2} \mathrm{O}_{2}$ is a strong oxidising agent and hence can increase the rate of oxidation substantially. Consequently, through $\mathrm{H}_{2} \mathrm{O}_{2}$ exposure, the effect of the surface composition on the oxide thickness was investigated. CZT samples were exposed to various $\mathrm{BM}$ concentrations and then dipped in $30 \% \mathrm{H}_{2} \mathrm{O}_{2}$ for 30 seconds. The oxide thickness is plotted as a function of BM concentration in Figure 5. There is a linear increase in the oxide thickness with BM concentration up to approximately $1.5 \%$ BM. Increasing the BM concentration from 2 to $5 \%$ resulted in only a small increase in oxide thickness.

The mechanically polished surface exhibited an oxide thickness of $1.4 \mathrm{~nm}$ and the $0.2 \% \mathrm{BM}$ etched sample a thickness of $1.6 \mathrm{~nm}$. The latter would suggest that the native oxide formed on this $0.2 \% \mathrm{BM}$ etched surface through air oxidation in Figure 4 , was tending to a 'limiting' thickness of $1.6 \mathrm{~nm}$. To examine if the $\mathrm{H}_{2} \mathrm{O}_{2}$ dipping time had any effect on oxide thickness, five $0.2 \% \mathrm{BM}$ etched surfaces were exposed in $30 \% \mathrm{H}_{2} \mathrm{O}_{2}$ for times varying between 1 and $300 \mathrm{~s}$. In all cases, the oxide thickness was determined to be $1.6 \pm 1.0 \mathrm{~nm}$ (16). It is clear that the $30 \% \mathrm{H}_{2} \mathrm{O}_{2}$ solution effects rapid oxidation of the CZT surface and even very short exposure times appear to result in oxide thicknesses similar to that eventually formed through air exposure.

Returning to Figure 5, the data shows that up to a BM concentration of approximately $1.5-2.0 \%$, as the thickness of the Te rich graded layer increases, a correspondingly thicker $\mathrm{TeO}_{2}$ layer forms when exposed to $\mathrm{H}_{2} \mathrm{O}_{2}$. This occurs due to an increasing amount of elemental $\mathrm{Te}$ being available at the surface to rapidly form the $\mathrm{TeO}_{2}$ layer. $\mathrm{BM}$ concentrations above $2 \%$ do not lead to a significant further thickening of the oxide, indicating that the Te enrichment in the CZT surface region has reached saturation and corresponds to a surface region which although probably still graded, is effectively pure Te. This is in good agreement with the ARXPS results given Fig. 3 (d) for a 5\% BM etch.

The incorporation of CZT crystals into detector assemblies requires surfaces which are defect free. The BM methanol treatment is known to be a simple and effective method for removing mechanical damage from the surface. In pixellated detectors, it is particularly important to establish CZT surfaces (away from the 
contacts) which are highly resistive, to reduce the leakage current and have long term stability in air. This study has shown how the surface chemistry of CZT can be easily controlled through simple $\mathrm{BM}$ etching and $\mathrm{H}_{2} \mathrm{O}_{2}$ passivation treatments. Further work is now required to correlate the different surfaces with the CZT detector performance.

\section{Conclusions}

(a) BM treatment of $\mathrm{Cd}_{0.95} \mathrm{Zn}_{0.05}$ Te gives rise to a graded Te-rich surface layer which increases in thickness as a function of BM concentration.

(b) Room temperature air oxidation of $0.2 \% \mathrm{BM}$ etched $\mathrm{Cd}_{0.95} \mathrm{Zn}_{0.05} \mathrm{Te}$ follows a logarithmic rate law, where the thickness of the $\mathrm{TeO}_{2}$ oxide layer, $d$ as a function of time, $t$ is described by the equation: $d=0.285 \ln (0.047 t+0.98)$.

(c) Exposure of $\mathrm{BM}$ treated $\mathrm{Cd}_{0.95} \mathrm{Zn}_{0.05}$ Te to $30 \% \mathrm{H}_{2} \mathrm{O}_{2}$ for 30 seconds shows a linear increase in the $\mathrm{TeO}_{2}$ oxide thickness with increasing $\mathrm{BM}$ concentration up to a BM concentration of $1.5 \%$. Above a $2 \%$ BM concentration, the Te enrichment in the CZT surface region has reached saturation and is effectively pure Te.

\section{Acknowledgements}

The authors would like to acknowledge the EPSRC for funding this work through the HEXITEC project.

\section{References:}

(1) James R et al., Semiconductors for Room Temperature Nuclear Detection Applications, Academic Press, New York, 1995, p. 384.

(2) Szeles Cs et al., 2007; IEEE Trans. Nucl. Sci., 54, 1350

(3) Bolotnikov A E et al, 2002; IEEE Trans Nucl.Sci. 49, 1942

(4) Chen $\mathrm{H}$ et al, J. Vac. Sci. Technology A, 1999, 17, 97

(5) Qiang L, Wangi J, Semicond. Sci. Technol. 2006, 21, 72

(6) Kowalsczyk S P, Cheung J T, J. Vac. Sci. Technology, 1981 18, 944 
(7) George M A, J. Appl. Physics 1995; 77, 7, 3134

(8) Chen K T et al, J. Vac Sci Technology A, 1997, 15, 850

(9) Rouse A A et al, IEEE Trans. Nuclear Sci. 2002, 49, 4

(10) Olego D J et.al. Applied Physics Letters, 1985, 47, 1172

(11) Watts J F and Wolstenholme J 'An Introduction to Surface Analysis by XPS and AES', 2003, Wiley, Chichester, UK, p83

(12) Seah M P, Dench W A, Surf. Interface Anal., 1979, 1, 2

(13) Etcheberry A et al J. Crystal Growth, 1998, 184/185, 213

(14) Bahl M K et al, J. Chem. Phys. 1977, 66, 5526

(15) Soriano L, Surf. Interface Anal., 1990, 16193

(16) Monnier M L T, MSc Thesis, 2009, University of Surrey, Guildford, Surrey, UK 
Figures;

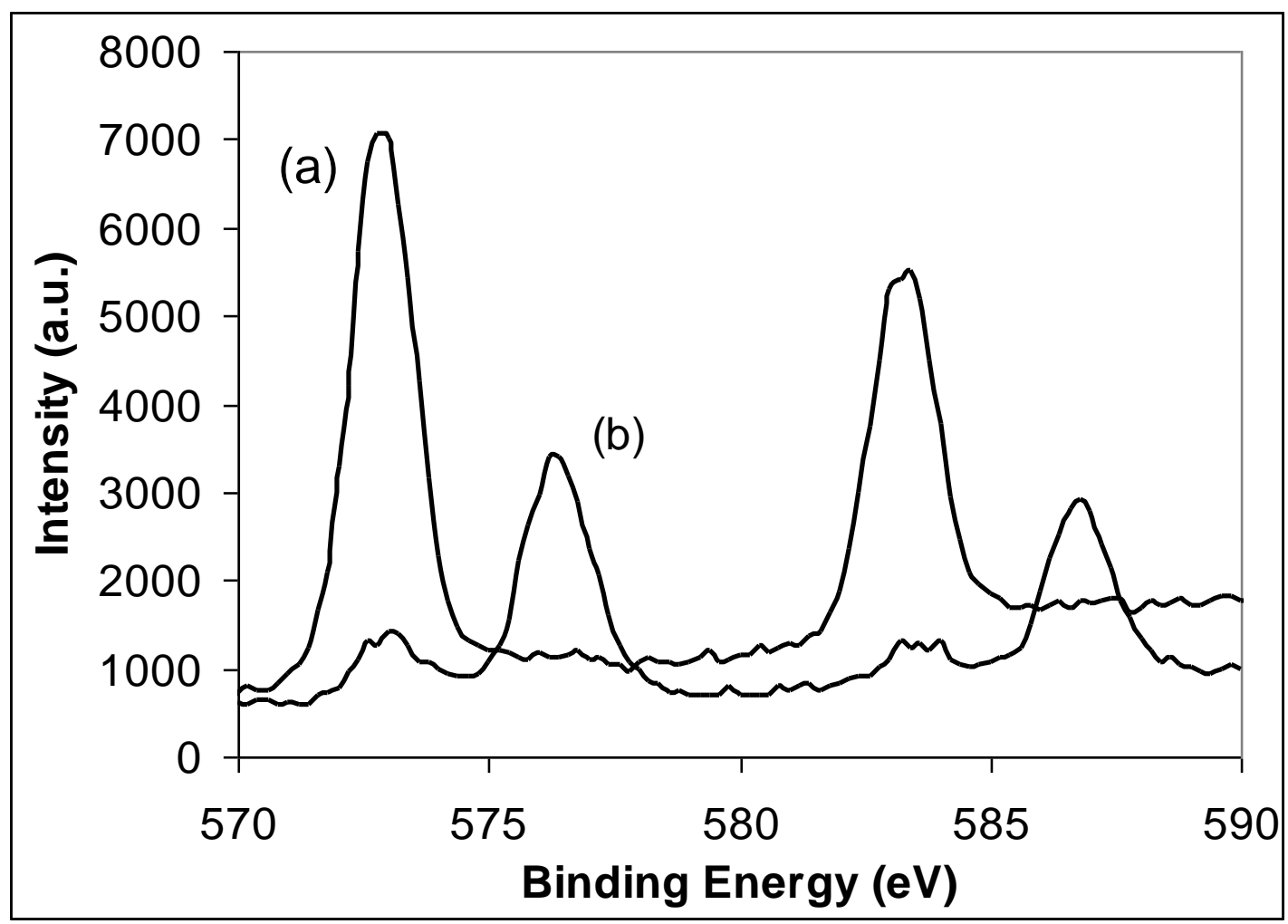

Figure 1. XPS Te 3d spectra for CZT surfaces: (a) Freshly etched in $2 \% \mathrm{BM}$; (b) Freshly etched in $2 \% \mathrm{BM}$ and subsequently oxidized by dipping into $\mathrm{H}_{2} \mathrm{O}_{2}$ for 2 mins. 

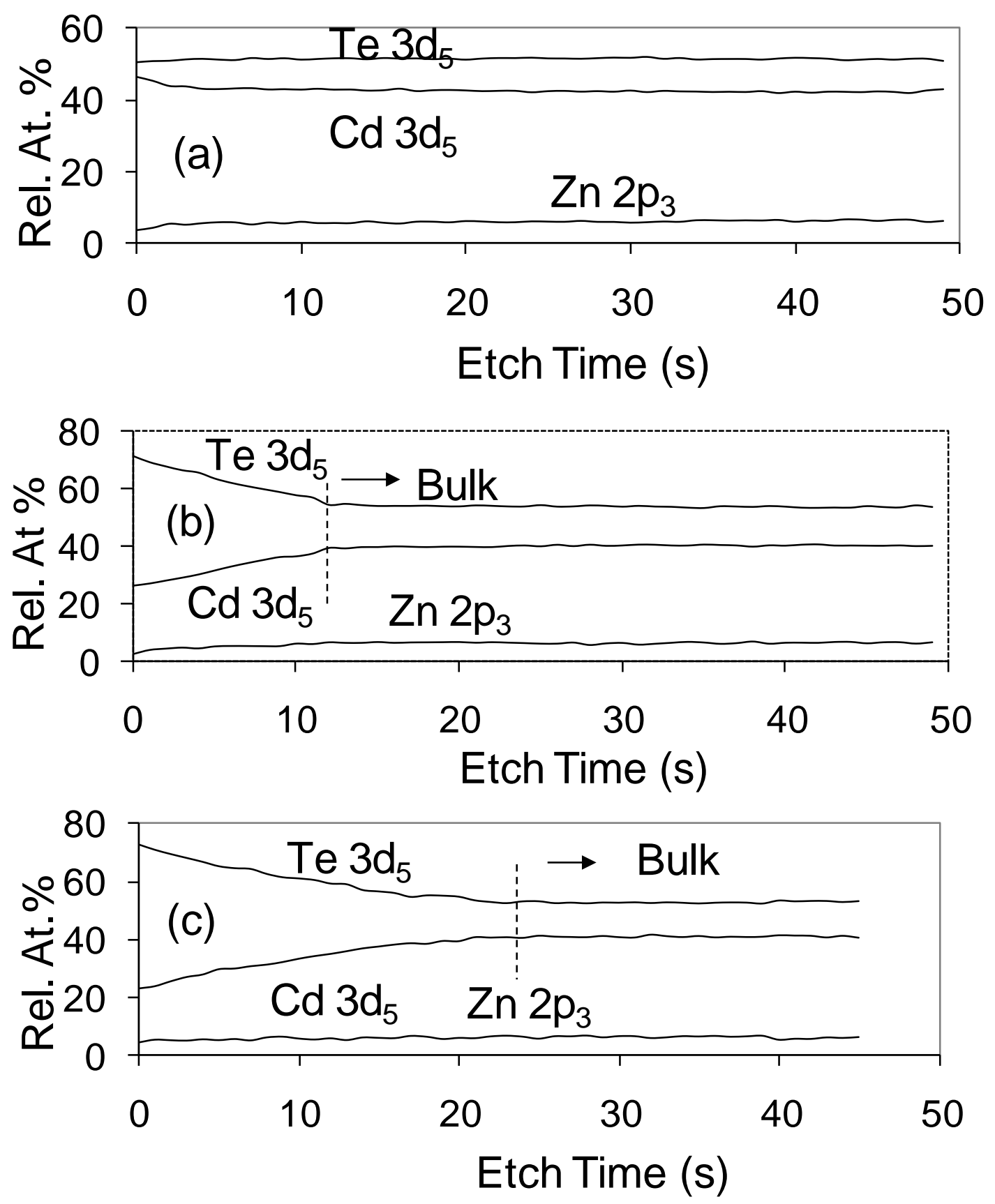

Fig. 2 XPS depth profiles of $\mathrm{Cd}_{0.9} \mathrm{Zn}_{0.1} \mathrm{Te}$ surface; (a) Mechanically polished only, (b) Etched in $0.2 \%$ BM for 2 mins, (c) Etched in $1.0 \%$ BM for 2 mins. 


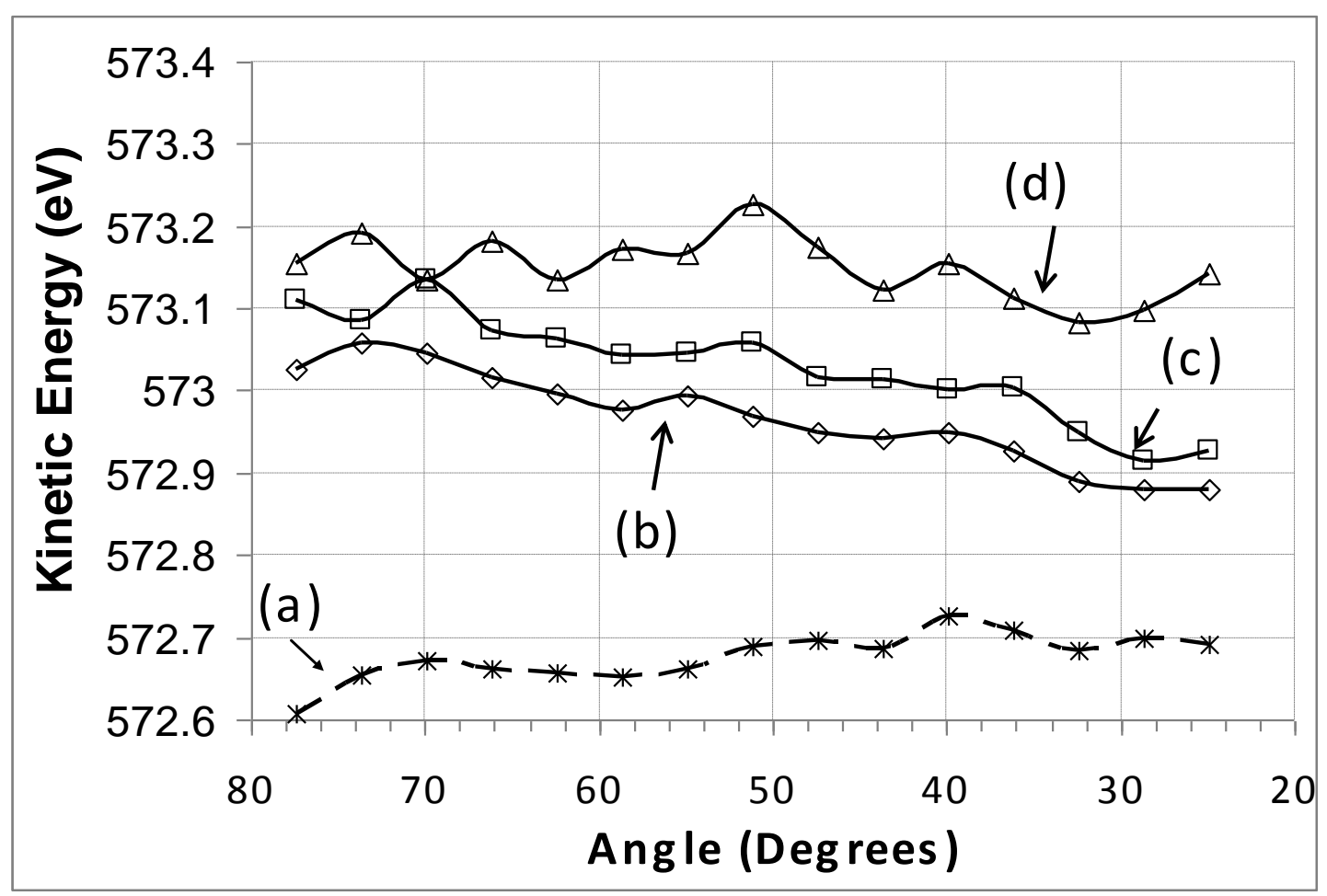

Fig.3 Angle resolved XPS showing the variation of $3 d_{5 / 2}$ Te (Met) peak energies for CZT sample surfaces that are (a) Cleaved; (b) $0.5 \%$ BM etched; (c) $1 \%$ BM etched; (d) $5 \%$ BM etched 


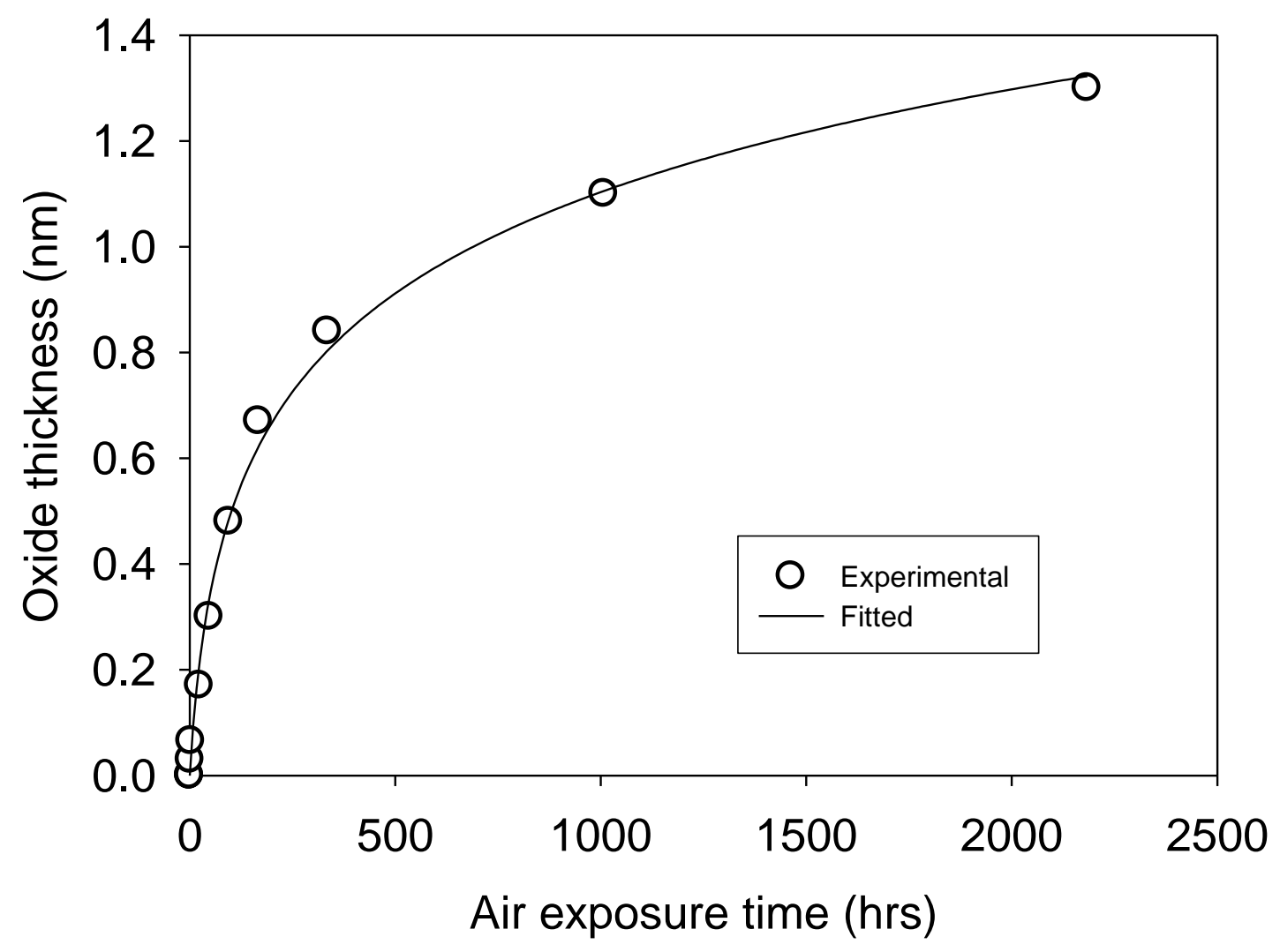

Fig.4. Air oxidation of a CZT surface after a $0.2 \%$ BM etch treatment. The experimental data has been fitted to the equation, $\boldsymbol{d}=0.285 \ln (0.047 \boldsymbol{t}+0.987)$, where $d$ is the oxide thickness and $t$, the exposure time. 


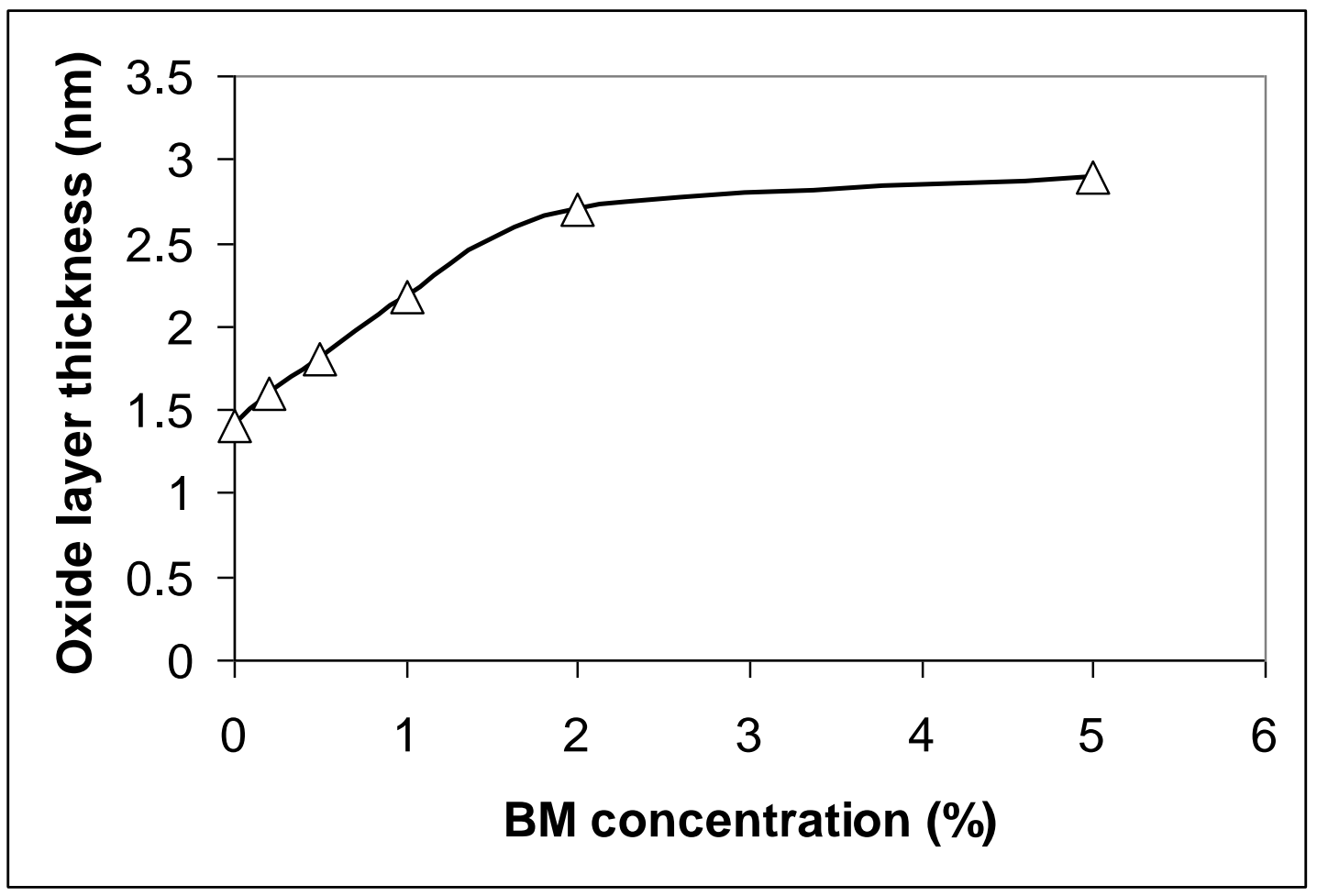

Fig.5 Effect of BM solution concentration on oxide thickness. 